\title{
Multivariate texture discrimination based on geodesics to class centroids on a generalized Gaussian manifold
}

\author{
A.Shabbir, G.Verdoolaege and G.Van Oost \\ Department of Applied Physics, Ghent University, \\ Sint-Pietersnieuwstraat 41, B-9000 Ghent, Belgium \\ aqsa.shabbiraugent.be
}

\begin{abstract}
A texture discrimination scheme is proposed wherein probability distributions are deployed on a probabilistic manifold for modeling the wavelet statistics of images. We consider the Rao geodesic distance (GD) to the class centroid for texture discrimination in various classification experiments. We compare the performance of GD to class centroid with the Euclidean distance in a similar context, both in terms of accuracy and computational complexity. Also, we compare our proposed classification scheme with the k-nearest neighbor algorithm. Univariate and multivariate Gaussian and Laplace distributions, as well as generalized Gaussian distributions with variable shape parameter are each evaluated as a statistical model for the wavelet coefficients. The GD to the centroid outperforms the Euclidean distance and yields superior discrimination compared to the k-nearest neighbor approach.
\end{abstract}

Keywords: Rao geodesic distance, texture discrimination, wavelet distributions.

\section{Introduction}

Variety of texture classification and retrieval techniques have been developed for tackling the issue of automated discrimination of textured images and their subsequent retrieval, both online and offline. The major challenge in this application is the classification and extraction of the desired image with maximized accuracy and least computational load.

Texture classification is essentially a two-stage process: feature extraction and similarity measurement. Feature extraction entails the extraction of a minimalist set of features that accurately depict the image in question. The subsequent similarity measurement requires the determination of a distance function which gauges the similarity of images on the basis of their respective feature sets. These two stages essentially dictate the design and performance of the classification and then the subsequent retrieval system.

Various popular and widely acknowledged texture discrimination techniques deploy filtering or wavelet-like approaches for accomplishing texture classification or retrieval, [1]. Essentially, these techniques make use of the enhanced ease of model- 
ing the information, when it is made available in a transformed domain. These approaches typically provide acceptable classification performances from large texture databases and are also endorsed by the physiological studies of the visual cortex which suggests that the wavelet decomposition is a natural way of image formation, [2]. Moreover, representation by wavelet features enables the classification schemes to operate directly in the compressed domain as wavelets is the principal technology in image coding formats like JPEG. These significant advantages and reasonable success of various wavelet based texture classification schemes, motivates our choice of wavelet representation of textures for this work.

In this study, we have exploited a parametric probabilistic framework for yielding a precise and accurate descriptor of images and thus obviating the need of storing or transmitting any redundant information. Numerous univariate models have been proposed for characterizing the wavelet subbands. Despite the ease of modeling and computation, these approaches do not completely exploit the rich texture information as they are inadequate for modeling the correlation between color bands. Multivariate distributions such as Generalized Gaussian [3], Gaussian Scale Mixture [4]and alphastable distributions [5] have, also, lately been utilized with varying degrees of success, for modeling the spatial and/or color correlations of the wavelet coefficients.

In this work, we employ a singular probabilistic model for modeling both the texture and color information, contained in the images. Verdoolaege et al. [3] established that classification and hence retrieval performance improves if the information contained in the correlation between color bands is exploited. Extending on this notion, in our probabilistic framework we utilize a multivariate probability distribution for joint modeling of the spectral bands while assuming independence amongst the wavelet subbands corresponding to the same color. In this work, we initially make use of the univariate Gaussian, Laplacian and generalized Gaussian distributions as our statistical model, and we then subsequently deploy the multivariate Gaussian, Laplacian and Generalized Gaussian distributions for comprehensive modeling of the rich correlation between color bands prevalent in the textured images.

Once feature extraction has been accomplished, determination of a suitable distance or similarity measure remains the next pursuit. As numerous possibilities exist in terms of probabilistic models which can be utilized for modeling the wavelet detail statistics, there is also a wide variety in terms of distance measures that can be used for evaluating the distance between probability distributions. Euclidean distance, despite yielding acceptable performances in various textural retrieval contexts [6], is not a natural similarity measure between probability distributions [3]. Kullback-Leibler divergence (KLD) despite its popularity for evaluating similarities is in fact not a true distance measure. The Rao geodesic distance (GD), derived from the Fisher information, has been used in case of multivariate probability distributions and has outperformed KLD and Euclidean in many contexts [3]. Furthermore, the GD is a natural similarity measure between probability distributions.

In this paper, we propose a new scheme for texture retrieval based on the calculation of the geodesic distance between the query image and the centroid of the texture classes. Furthermore, to provide an ease of reference, we compare the performance of our proposed scheme with the performance of the k-nearest neighbour classifier using 
the Euclidean distance. We also evaluate the outcomes of our proposed technique when it operates with Euclidean distance as the underlying distance measure. Initially we work with the grey-level textures generated from the luminance of the RGB colour images and we then move on to full joint modelling of the wavelet coefficients corresponding to the three colour bands. We also examine the computational expense of our proposed classification technique. The rest of the paper is organised as follows. Section 2 summarises the statistical models, the Rao geodesic distance and our proposed texture classification scheme. Section 3 outlines the experimental setup and presents the attained classification results. Finally, Section 4 concludes the paper.

\section{$2 \quad$ Statistical modeling and similarity measures}

\section{1 (Multivariate) generalized Gaussian distribution}

The multivariate generalized Gaussian distribution has been introduced in [7] for modeling the wavelet detail co-efficients. We present the univariate generalized Gaussian distribution, before proceeding to the multivariate case. The univariate generalized Gaussian distribution is given as:

$$
f(x \mid \alpha, \beta)=\frac{\beta}{2 \alpha \Gamma[1 / \beta]} \exp \left[-\left(\frac{|x|}{\alpha}\right)^{\beta}\right]
$$

Where $\Gamma$ (.) denotes the Gamma function and $\alpha$ and $\beta$ are, respectively, the scale and shape parameter controlling the variance and the fall-off rate of the distribution. $\beta=2$ yields the Gaussian distribution and $\beta=1$, results in the Laplace distribution. We proceed to the multivariate generalized Gaussian distribution, defined in [4] as:

$$
f(\mathbf{X} \mid \Sigma, \beta)=\frac{\Gamma \frac{m}{2}}{\pi^{\frac{m}{2}} \Gamma\left(\frac{m}{2 \beta}\right) 2^{\frac{m}{2 \beta}}} \frac{\beta}{|\Sigma|^{\frac{1}{2}}} \times \exp \left\{-\frac{1}{2}\left[\mathbf{X}^{\prime} \Sigma^{-1} \mathbf{X}\right]^{\beta}\right\}
$$

Here, $\mathrm{m}$ is the dimensionality of the probability space, and is equal to 3 in our case of colored images. The distribution reduces to a multivariate Gaussian case for $\beta=$ 1and to a multivariate Laplace case for $\beta=0.5$. $\sum$ is the dispersion matrix. Parameters for multivariate MGGD, Laplace and Gaussian were estimated using the method of moments, followed by maximum likelihood estimation [3].

\subsection{Geodesic distance}

The Rao geodesic distance in the context of information geometry provides an effective distance measure between probability distributions represented by points on a probabilistic manifold. Geodesic distances allow for length minimization on the prob- 
abilistic manifold and offer an edge in terms of data visualization that they enable on the manifold [8].

For fixed shape parameter $\beta$ i.e. Laplace and Gaussian case, the geodesic distance between two MGGDs denoted by $\left(\beta, \Sigma_{1}\right)$ and $\left(\beta, \Sigma_{2}\right)$ is given as [3]:

$$
G D=\left[\left(3 b_{h}-\frac{1}{4}\right) \sum_{i}\left(r_{2}^{i}\right)^{2}+2\left(b_{h}-\frac{1}{4}\right) \sum_{i<j} r_{2}^{i} r_{2}^{j}\right]^{\frac{1}{2}}
$$

Here, $r_{2}^{i} \equiv \ln \lambda_{2}^{i}$ and $\lambda_{2}^{i}, i=1, \ldots, m$, the $m$ eigenvalues of $\Sigma_{1}^{-1} \Sigma_{2}$. Also, $b_{h}$ is defined by

$$
b_{h} \equiv \frac{1}{4} \frac{m+2 \beta}{m+2}
$$

With variable shape parameter there is no closed form for the GD and we used a linear approximation to the geodesic coordinate functions, to render the calculations computationally more feasible, see [9].

\subsection{Distance-to-centroid classifier}

We present a novel classification scheme for data points (i.e. textures in this application) expressed as probability distributions and laying as points on a probabilistic manifold. The scheme is outlined as:

- Training data is used for computing the centroid for each class of textured images. The geodesic centroid is calculated according to an iterative algorithm described in [12], based on a projection on the tangent space. To realize this, the (inverse) exponential map was calculated for each of the distribution models used in this work.

- Distance is evaluated between the class centroids and each test data object (query image in this case), which is to be classified.

- Geodesic distance is used as the distance measure, due to its suitability as a natural distance measure between probability distributions.

- Test data object is assigned the class, whose centroid has the shortest geodesic distance to the object.

\section{Classification Experiments}

\subsection{Experimental setup}

We carried out our experiments with grey-level and colored textures from a small dataset of 40 images from the Vistex database [11]. This is the same database that was used by Verdoolaege et al. [3] and Do et al. [6] for conducting wavelet-based texture retrieval. This enables a comparison with their results in the similar context. The da- 
tabase comprises of glimpses of different real-world natural scenes possessing sufficient homogeneity and having a $512 \times 512$ image size. Each image was divided into 16 128 x 128-sized non-overlapping subimages, yielding a database of 640 subimages. Furthermore, each subimage was expressed in the RGB color space. Grey-level images were generated from the original color images by calculating their luminance. Moreover, every color (or grey-level) component of each subimage was individually normalized to zero mean and unit variance resulting in the subimages from the same original image not generally lying in the same range, rendering the problem more challenging. Following this, a discrete wavelet transform was applied on every component with three levels using the Daubechies filters of length eight. The wavelet detail coefficients of every subband over the three color components (or the greylevel) were modeled by a (multivariate) Gaussian or Laplace distribution, or a generalized Gaussian distribution with variable shape parameter. The parameters of the probability models for all subbands constitute the feature set for a single subimage.

The classification experiment was implemented in two stages: training and testing. In the training stage, the class label of each image was assumed to be known, which enabled the calculation of a centroid for each class. In the testing phase, the distance between the test image and the centroid of each class was calculated. The test image was then assigned the class, whose centroid had the smallest distance to the test image. Following that, we compared the assigned class label with the actual class label of the test image. We carried out the experiment repeatedly, using every subimage as a test image once. We finally calculated the average rate of successful classification as a performance measure.

The experiments were conducted with the geodesic distance as a distance measure between the test image and the class centroid, and subsequently using the Euclidean distance. This way the GD could be compared as a similarity measure between probability distributions to the Euclidean distance.

In the last stage, the classification was also performed using the k-nearest neighbor classifier in conjunction with the Euclidean distance, to provide a reference for comparison of our proposed technique. When working with the k-nearest neighbor algorithm, we considered one of the 640 subimages to be a test image which is to be assigned to one of the 40 classes. The class labels of the other subimages were assumed to be known. Distance between the test image and each of the remaining images was determined and the test image was assigned to the class most common among the fifteen nearest neighbours of the test image. Choice of fifteen nearest neighbours is motivated by the hypothesis that the fifteen nearest neighbours of the test image should be the fifteen subimages originating from the same class to which the test image belonged. Following that, we compared the assigned class label with the actual class label of the test image. Again, we carried out the experiment repeatedly, using every subimage as a test image once. The correct classification rate was then assessed by calculating the ratio of the images that were correctly classified to the total number of images. 
We conducted the classification experiments initially on the grey-level equivalent of the 640 colour images and then we catered the corresponding full RGB colour images considering the complete correlation structure between the spectral bands. For each of these instances, as a statistical model for wavelet coefficients, we employed the multivariate Gaussian, Laplacian and generalized Gaussian, characterised by $\beta=1, \beta=1 / 2$ and variable $\beta$, respectively.

\subsection{Computational demands}

Besides accuracy, computational load of a retrieval or classification technique is also a crucial yardstick of performance. Computational efficiency has a direct impact on the required resources and speed, and can be a limiting factor in various applications. We have measured the time taken by our proposed technique to classify a query image based on the distance to the class centroids and also the time taken for the k nearest neighbor algorithm to perform the same feat. The time taken for computation was measured on the same machine on which all calculations pertaining to this work were performed. The machine employed for this work was a Dell Precision T7600 equipped with an Intel Xenon(R) CPU at $2.4 \mathrm{GHz}$ and $16 \mathrm{~GB}$ of RAM, running the 64-bit version of the Windows 7 operating system. The retrieval systems were implemented and run in MATLAB (version 8, R2012b, 64 bit) [12]. The durations are presented in Table 1.

\begin{tabular}{|c|c|c|c|c|}
\hline Classifier & Measure & Model & Gray Images & Colour Images \\
\hline \multirow{3}{*}{$\begin{array}{c}\text { Distance-to- } \\
\text { centroid }\end{array}$} & \multirow{3}{*}{ Geodesic } & Gauss & 0.040 & 0.300 \\
\cline { 3 - 5 } & & Laplace & 0.042 & 0.330 \\
\cline { 3 - 5 } & \multirow{2}{*}{ Euclidean } & GGD & 0.476 & 1.301 \\
\cline { 3 - 5 } & & Gauss & 0.015 & 0.043 \\
\cline { 3 - 5 } & & Laplace & 0.015 & 0.044 \\
\cline { 3 - 5 } & & GGD & 0.034 & 0.094 \\
\hline \multirow{2}{*}{$\begin{array}{c}\text { k-Nearest } \\
\text { Neighbour }\end{array}$} & \multirow{3}{*}{ Euclidean } & Gauss & 0.241 & 0.690 \\
\cline { 3 - 5 } & & Laplace & 0.242 & 0.700 \\
\cline { 3 - 5 } & & GGD & 0.550 & 1.500 \\
\hline
\end{tabular}

Table 1. Time, in ms, necessary for the classification of one textured image (nine wavelet subbands), using geodesic distance to centroid classifier and the kNN classifier, characterized by different models.

Classification conducted with the distance-to-centroid classifier, employing GD as the distance measure, takes considerably longer than the same classifier working with Euclidean measure. However, this is a direct consequence of accuracy-versus-speed tradeoff, as the classification accuracy with GD clearly outperforms Euclidean with a large margin. The most noteworthy observation is the superior performance of our proposed distance-to-centroid classifier, compared to a k-nearest neighbour classifier, in terms of computational load. Distance-to-centroid proves to be computationally 
attractive, as it essentially reduces the comparisons required to correctly classify one query image, to the number of centroids, which are equal to the number of classes. knearest neighbour, ideally, requires $\mathrm{N}$ number of comparisons to accomplish the same task, where $\mathrm{N}$ is the number of entries in the database. Laplace and Gaussian models consume less time for geodesic distances, in contrast to generalized Gaussian, which is a direct repercussion of the necessity of approximating the GD in the latter case.

\subsection{Results and discussion}

The results of our classification experiments on the Vistex database are presented in Table 2, followed by a discussion on significant observations.

\begin{tabular}{|c|c|c|c|c|}
\hline Classifier & Measure & Model & Grey Images & Colour Images \\
\hline \multirow{3}{*}{$\begin{array}{c}\text { Distance-to- } \\
\text { Centroid }\end{array}$} & \multirow{3}{*}{ Geodesic } & Gauss & 83.59 & 97.17 \\
\cline { 3 - 5 } & & Laplace & 84.38 & 97.81 \\
\cline { 3 - 5 } & \multirow{2}{*}{ Euclidean } & GGD & 87.19 & 97.19 \\
\cline { 3 - 5 } & & Gauss & 46.10 & 58.91 \\
\cline { 3 - 5 } & & Laplace & 45.63 & 58.91 \\
\cline { 3 - 5 } & & GGD & 42.03 & 50.00 \\
\hline \multirow{2}{*}{$\begin{array}{c}\text { K-Nearest } \\
\text { Neighbour }\end{array}$} & \multirow{2}{*}{-} & Gauss & 67.50 & 78.13 \\
\cline { 3 - 5 } & & Laplace & 65.16 & 77.03 \\
\cline { 3 - 5 } & & GGD & 55.47 & 47.97 \\
\hline
\end{tabular}

Table 2. Correct classification rates(\%), using different models for three wavelet scales, using distance to centroid and k-nearest neighbour classifiers.

It can be observed that when the correlation structure between the spectral bands is considered (i.e colour images), the classification accuracy is substantially enhanced in comparison to grey scale modeling. This however, happens at an escalated computational expense. The most significant result is the high classification accuracy, achieved with our proposed distance-to-centroid classifier based on GD, in contrast to the k-nearest neighbour classifier. Superior performance of distance-to-centroid classifier with GD, as opposed to Euclidean, further substantiates the worth of the GD as a well suited distance measure for probability distributions on a manifold. Finally, the GGD yields higher classification accuracy for grey-scale images with the GD-based distance-to-centroid classifier.

\section{Conclusion and future work}

In this paper we have proposed a new technique for classifying textures, when they are represented in the wavelet domain. We have shown the value of the Rao geodesic distance as an efficient distance measure between probability distributions and hence, 
as an important aid to effective classification. We have also illustrated how texture classification can profit by exploiting the information residing in the rich spectral band correlation structure by joint modeling through multivariate distributions. Furthermore, we have applied various statistical models and hence we have showed their respective competences for accomplishing the task.

In the future, we envisage investigating the behavior of our developed technique and obtained conclusions on other data sets and applications. Furthermore, we plan to improve our classification technique by incorporating class variance, leading to the calculation of Mahalanobis distances on tangent spaces. Analyzing the effect of additive noise on the performance of our classifier is also aspired.

Acknowledgements. This work was supported by the European Commission and carried out within the framework of the Erasmus Mundus International Doctoral College in Fusion Science and Engineering (FUSION-DC).

\section{References}

1. A. Laine and J. Fan, "Texture classification by wavelet packet signatures," IEEE Trans. Pattern Recognit. Machine Intell., vol. 15, pp. 1186-1191, 1993.

2. J. Daugman, "Two-dimensional spectral analysis of cortical receptive field profile," Vision Research, Vol.20 (10), pp. 847-856, 1980.

3. G.Verdoolaege and P.Scheunders, "Geodesics on the manifold of multivariate generalised Gaussian distributions with an application to multicomponent texture discrimination," International Journal of Computer Vision, Vol. 95, pp. 265-285, 2011.

4. P.Scheunders, and S. De Backer, "Wavelet de noising of multicomponent images, using Gaussian Scale Mixture models and a noise-free image as priors," IEEE Transactions on Image Processing, Vol. 16, pp. 1865-1872, 2007.

5. G.Tzagkarakis, B. Beferull-Lozano, and P.Tsakalides, "Rotation-invariant texture retrieval with gaussianized steerable pyramids," IEEE Transactions on Image Processing, Vol. 15, pp. 2702-2718, 2006.

6. M. Do and M. Vetterli, "Wavelet-based texture retrieval using generalized Gaussian density and Kullback-Leibler distance," IEEE Transactions on Image Processing, Vol. 11, pp. 146-158, 2002.

7. G. Verdoolaege, S. De Backer, and P. Scheunders, "Multiscale Colour Texture Retrieval using the Geodesic Distance between Multivariate Generalized Gaussian Models," in IEEE International Conference on Image Processing, 2008, pp. 169-172.

8. G Verdoolaege, G Karagounis, M Tendler and G Van Oost, "Pattern recognition in probability spaces for visualization and identification of plasma confinement regimes and confinement time scaling," Plasma physics and controlled fusion, Vol. 52, No.12, 2012.

9. G.Verdoolaege and P.Scheunders, "On the geometry of multivariate generalised Gaussian models," Journal of mathematical imaging and vision, 2011.

10. X. Pennec, P. Fillard and N. Ayache, "A Riemannian framework for tensor computing," International Journal of Computer Vision, Vol. 66, pp. 41-66, 2006.

11. MIT vision and modeling group (2010). Vision texture. Online at http://vismod.media.mit.edu/vismod/imagery/VisionTexture/.

12. The Mathworks (2012). Natick, MA. www.mathworks.com. 